\title{
Transformation des Subjekts im Kontext des Übergangs in die Hochschule
}

Der vorliegende Beitrag beschäftigt sich aus der Sicht des Subjekts mit Transformationen und nimmt die dabei (potentiell) stattfindenden Bildungsprozesse in den Blick. Diese werden im spezifischen Kontext des Übergangs in die Hochschule empirisch analysiert. Durch diesen Fokus wird es möglich, unterschiedliche Facetten von transformatorischer Bildung sowie Hinweise auf selbstbezogene Veränderungen in den Eigentheorien der Subjekte herauszuarbeiten. Darüber hinaus lässt sich die Funktion dieses spezifischen Übergangs als Generator solcher Bildungsprozesse rekonstruieren.

Vor diesem Hintergrund werden folgende Fragestellungen bearbeitet, die über ihren Bezug zur subjektiven Gestaltung des Übergangs miteinander verbunden sind:

- Welche Dimensionen charakterisieren den Übergang in die Hochschule aus Sicht der Betroffenen?

- Welche selbstbezogenen Veränderungen lassen sich in den Eigentheorien der Befragten identifizieren?

Um einen Beitrag zur Diskussion dieser Fragestellungen zu leisten, wird zunächst das Forschungsfeld der Hochschullandschaft dargestellt. Nach der Rahmung der zentralen theoretischen Konzepte wird das Forschungsdesign erläutert, auf dessen Ergebnissen der vorliegende Beitrag beruht. Dem schließen sich die zentralen empirischen Ergebnisse sowie deren Rückkopplung an den Forschungsstand an.

\section{Veränderungen in der Hochschullandschaft}

Wie jede pädagogische Organisationsform hat auch die Hochschule im historischen Verlauf vielfältige Veränderungen erfahren. Dies betrifft bspw. nicht nur die Ausdifferenzierung in verschiedene Institutionalisierungsformen, sondern auch die Zielsetzungen, mit denen der Besuch von Hochschulen verbunden war und ist (Fisch 2015). Auch der Blick auf die jüngere Geschichte der Hochschule offenbart substantielle Veränderungen, die anhand unterschiedlicher Phänomene sichtbar werden. 
Als eines dieser Phänomene kann die angebotsseitige Ausdifferenzierung von Hochschulen identifiziert werden. Exemplarisch wird dies anhand der Anzahl der Studiengänge in Deutschland deutlich. So stieg ihre Anzahl in den letzten zehn Jahren um etwas mehr als 59\% auf insgesamt 19.011 derartiger hochschulischer Angebote (Hochschulrektorenkonferenz 2017: 9). Die angesprochenen Veränderungen zeigen sich darüber hinaus im Anstieg der totalen Studierendenzahlen. Betrachtet man die Entwicklung seit dem Jahr 2000, so lässt sich auch hier ein deutlicher Anstieg um knapp 62\% auf 2.844.978 Personen verzeichnen. Eine besondere Rolle unter den verschiedenen Institutionalisierungsformen der Hochschulen spielen hierbei die Fachhochschulen. Deren Anteil an der Gesamtzahl der Studierenden in Deutschland stieg zwischen dem Wintersemester 1999/2000 und dem Wintersemester 2017/2018 von rund 24\% auf knapp 35\% an (Statistisches Bundesamt 2018a: 13). Jenseits des genuinen Anstiegs der Studierendenzahlen zeigt sich eine deutliche Erhöhung der Studienanfänger*innenquote. Je nach Berechnungsweise ist der Anteil der Studienanfänger*innen an der Bevölkerung des entsprechenden Geburtsjahres zwischen den Jahren 2000 und 2017 von etwa 33\% auf 56\% angestiegen (Statistisches Bundesamt 2018b).

Neben dem angebotsseitigen Ausbau der Hochschullandschaft und einer steigenden Nachfrage durch eine Erhöhung der Studierendenzahlen bzw. der entsprechenden Quote lassen sich die Veränderungen in der Hochschullandschaft auch anhand der Zusammensetzung der Studierendenschaft erkennen. Dies wird anhand zweier Kennzahlen deutlich. Erstens sind hier die berufsbiografischen Vorerfahrungen zu nennen: Inzwischen verfügen $22 \%$ der Studienanfänger*innen über eine abgeschlossene Berufsausbildung (Autorengruppe Bildungsberichterstattung 2018: 157). Dies führt unter anderem auch zu einer Erhöhung des Lebensalters von beruflich Qualifizierten zu Studienbeginn (Middendorf et al. 2017: 29). Zweitens wird dieses Phänomen auch anhand der zuvor erworbenen Zugangsberechtigungen sichtbar. Nach wie vor bildet die allgemeine Hochschulreife den klassischen Zugang in die Hochschule, doch zeigt sich beispielsweise anhand der zunehmenden Nutzung des Dritten Bildungswegs eine Tendenz der Öffnung gegenüber anderen Wegen in diesen Bereich des Bildungssystems. Im Vergleich zum Jahr 2000 haben 2017 bereits siebenmal mehr Personen diesen Zugangsweg in Hochschulen gewählt, wobei anzumerken ist, dass ihr Anteil an den Studienanfänger*innen mit 3\% weiterhin eher überschaubar ausfällt (Autorengruppe Bildungsberichterstattung 2018: 157).

Insgesamt lässt sich festhalten, dass die Hochschullandschaft, exemplarisch dargestellt anhand ihrer Entwicklungen seit dem Jahr 2000, hinsichtlich ihrer Angebote von expansiven Tendenzen geprägt ist. Ihre Angebote werden von immer mehr Personen nachgefragt und im Hinblick auf die Studierendenschaft lässt sich zudem eine Pluralisierung diagnostizieren. Vor diesem Hin- 
tergrund eröffnen sich mehr Möglichkeiten im Erwachsenenalter, um Hochschulen als formale Lernkontexte zu nutzen. Der vorliegende Beitrag thematisiert in diesem Zusammenhang die subjektiven Veränderungen der Studierenden im Rahmen der Aufnahme eines Hochschulstudiums, wobei der Übergang in die Hochschule als potentiell kritisches Lebensereignis verstanden wird.

\section{2 Übergangs- und Transformationsprozesse}

$\mathrm{Zu}$ den unterschiedlichen Facetten von Übergängen können theoretische Bezüge hergestellt werden, die je eigene Schwerpunkte auf derartige Prozesse legen. Im vorliegenden Beitrag wird das Konzept der Life-Trajectory (Schäffter 2015) zur Rahmung von Übergängen gewählt, das sich aufgrund verschiedener Aspekte für die Analyse von biografischen Prozessen besonders eignet: Aus dieser Perspektive heraus werden Übergänge im Sinn einer Life-Trajectory zunächst als soziale Prozesse verstanden, die jedoch nicht primär durch institutionalisierte Rahmungen strukturiert, sondern vielmehr ohne eine solche Steuerungsinstanz zu denken sind. Der Prozesscharakter wird in diesem Konzept zudem damit unterstrichen, dass Übergänge als eine pfadabhängige Abfolge von einzelnen Lebensereignissen verstanden werden, was die Prognosemöglichkeit für den Eintritt spezifischer Ereignisse erschwert (Schäffter 2015: 27f.). Gleichzeitig werden dadurch Phänomene vielfältiger Verstrickungen und Verknüpfungen zwischen Lebensereignissen, beispielsweise zwischen privaten und beruflichen Entwicklungen oder persönlichen und familienbiografischen Erfahrungen, theoretisch aufgegriffen und empirisch zugänglich. Ein weiteres Kennzeichen der Life-Trajectory, das für die Analyse von biografischen Veränderungen und vor allem den damit verbundenen Bildungsprozessen relevant ist, bildet die Zieloffenheit dieses Zugangs. Die damit verbundene prospektive Ausrichtung verweist auf einen Möglichkeitsraum für zukünftige Entwicklungen, der dem Subjekt einen multioptionalen Spielraum zur Gestaltung der eigenen Übergangsprozesse und damit des eigenen Lebensverlaufs ermöglicht. Um die entsprechenden Übergänge zu realisieren, wird im vorgestellten Konzept auch eine zentrale Bedingung an das Subjekt formuliert. Um beispielsweise Kontexterschließung zu ermöglichen, wie sie bei der Aufnahme eines Hochschulstudiums im Sinn einer transformativen Aneignung von Lernkontexten nötig wird, bedarf es vonseiten des Subjekts einer permanenten Selbstvergewisserung über die Zieloffenheit des eigenen Handelns (Schäffter, 2015: 29).

Der im Zusammenhang von Life-Trajectory bereits am Beispiel der transformativen Aneignung von Lernkontexten angesprochene Aspekt der Trans- 
formation findet sich in einem weiteren anschlussfähigen Konzept. Dessen Relevanz ergibt sich vor dem Hintergrund der Diagnose einer Transformationsgesellschaft (Schäffter 2014: 37) und den damit korrespondierenden Auswirkungen auf die Anforderungsstrukturen und Möglichkeitsräume der subjektiven Lebensführung. Daran anschließend lässt sich das Konzept der transformatorischen Bildungsprozesse (Koller 2018) vor allem hinsichtlich zweier Facetten für die Analyse von Übergängen in die Hochschule nutzbar machen. Erstens wird Bildung aus dieser Perspektive als „Prozess der Transformation grundlegender Figuren des Selbst- und Weltverhältnisses“ (Koller 2018: 17) verstanden, wodurch die Auswirkungen hochschulischer Lernkontexte jenseits von Wissensvermittlungs- und -aneignungsprozessen fassbar werden. Zweitens lassen sich aus dieser Sicht Übergänge als Krisen greifen, die als Anlässe zur Transformation durch Infragestellung etablierter Welt- und Selbstbezüge verstanden werden können.

Die zwei vorgestellten Bezüge ermöglichen die Analyse von selbstbezogenen Veränderungen, die Subjekte im Kontext des Übergangs in die Hochschule erleben. Um diese Erfahrungen empirisch zu erfassen, wurde ein spezifischer Zugang zum Forschungsfeld gewählt, der die Eigentheorien der Subjekte in den Mittelpunkt rückt.

\section{Erforschung der Wege in die Hochschule}

Um die oben formulierten Fragestellungen vor dem Hintergrund empirischer Ergebnisse diskutieren zu können, wurde ein monomethodisches und explorativ-rekonstruktives Design gewählt. Im Fokus stehen dabei die verschiedenen Phänomene des Übergangsprozesses, der von den befragten Subjekten im Kontext ihrer Aufnahme eines Hochschulstudiums durchlaufen wird. Als Studienteilnehmende wurden dabei studienberechtigte Personen, eingeschriebene Studierende, exmatrikulierte Personen sowie Absolvent*innen von hochschulischen Studiengängen in Betracht gezogen. Der Forschungsprozess beruht auf den method(olog)ischen Prämissen der Grounded Theory, was sich vor allem in Bezug auf das Sampling sowie die Verwobenheit von Datenerhebung, -analyse und Theoriebildung auswirkt (Strübing 2014: 9ff.). Die Datenerhebung geschah durch problemzentrierte Interviews (Witzel/Reiter 2012: 15), sodass einerseits der thematische Fokus des Gesprächs mittels eines Leitfadens gerahmt und andererseits die Relevanzsetzungen der Befragten zur Geltung kommen konnten. Um die interessierenden Phänomene zu rekonstruieren, wurde auf das theoretische Kodieren der Grounded Theory zurückgegriffen (Strübing 
2014: 15f.). Dadurch wird auch auf der Ebene der Datenauswertung gewährleistet, dass die Perspektivität der Subjekte auf den eigenen Übergang in die Hochschule mit in den Prozess der Theoriebildung einfließt.

Die nachfolgend dargestellten Ergebnisse basieren auf einem kontrastiv ausgewählten Bestandteil des Samples. Es wurden drei Interviews mit Personen ausgewählt, die sich hinsichtlich ihrer Generationslagerung, des Geschlechts, der gewählten Studiengänge, der Studiengestaltung hinsichtlich der dabei realisierten Hochschulwechsel sowie der jeweiligen Studienphase unterscheiden. Diese Fälle werden im Rahmen der Ergebnisdarstellung als P1, P2 und $\mathrm{P} 3$ bezeichnet.

\section{Empirische Ergebnisse}

Aufgrund der geringen Fallzahl und des damit korrespondierenden Stands der Theoriebildung sind die nachfolgenden Ergebnisse als noch vorläufig zu betrachten. Trotz dieses frühen Auswertungsstadiums lassen sich jedoch bereits einige zentrale Facetten beschreiben, die sich mit Blick auf die oben ausgeführten Fragestellungen als instruktiv erweisen. Die mit ihnen korrespondierenden Resultate werden im Folgenden nach den beiden Fragestellungen getrennt dargestellt.

\section{Konturierung des Übergangs in die Hochschule}

Im Hinblick auf die thematisierten Dimensionen des Übergangs in die Hochschule aus Sicht der Betroffenen kann zunächst der multioptionale Spielraum zur Gestaltung der eigenen Übergangsprozesse konturiert werden. Bei der damit verbundenen Frage, in welchen Kontexten der Übergang realisiert wird, verweisen die Studienteilnehmenden auf den Umstand, dass neben der Aufnahme eines Hochschulstudiums weitere Übergangsprozesse vollzogen werden. In diesem Kontext verorten sie den Wohnortwechsel, die Aufnahme einer Erwerbstätigkeit, den Zusammenzug mit der*dem Partner*in, die Familiengründung sowie die damit nicht zwangsläufig verbundene Kindererziehung. Bei genauerer Betrachtung dieser Konstellationen fällt zudem die Präsenz simultaner Übergänge im Erwachsenenalter (Hof/Maier-Gutheil 2014: 147) ins Auge. Exemplarisch lässt sich dies anhand der Angaben von P2 beschreiben: Diese Person verbindet die Aufnahme eines Hochschulstudiums mit einem Wechsel des Wohnorts in ein anderes Bundesland und zieht simultan dazu mit 
ihrer*ihrem Partner*in zusammen. Dieses Phänomen zeigt nicht nur die Vielschichtigkeit des vollzogenen Übergangsprozesses auf, sondern verweist auf zwei weitere Aspekte: Erstens verdeutlicht die erläuterte Simultanität das Potential empirischer Analysen für die Erweiterung theoretischer Perspektiven auf Übergangsprozesse. Der bereits in Beschreibungen von schrittweisen Übergängen oder auch Ereignisketten aufscheinende Fokus auf Sequenzialität lässt sich vor dem Hintergrund der empirischen Ergebnisse zumindest kritisch hinterfragen. Zweitens leitet sich aus der Diagnose simultaner Übergänge eine potentiell steigende Komplexität der Anforderungsstrukturen für das Subjekt ab. Je nach Lebenssituation und Ressourcen erhöht sich dabei die Wahrscheinlichkeit für die Einstufung der Konstellation als Krise (Koller 2018: 71ff.).

\section{Entscheidung für die Aufnahme eines Hochschulstudiums}

Fragt man vor dem Hintergrund des Prozesscharakters von Übergängen in die Hochschule nach dessen Beginn, so wird im empirischen Material die Studienentscheidung in den Vordergrund der Sachverhaltsdarstellungen gerückt. Es zeigt sich in diesem Zusammenhang einerseits, dass der konkrete Zeitpunkt zur Aufnahme eines Hochschulstudiums nach dem Erwerb der Hochschulzugangsberechtigung variabel ausgestaltet wird. So entscheiden sich manche Personen für einen mehrmonatigen Auslandsaufenthalt, während andere einen unmittelbaren Anschluss nach dem Verlassen der Institution Schule anstreben. Allen Fällen gemeinsam ist andererseits die Kopplung der grundsätzlichen Entscheidung für die Aufnahme eines Hochschulstudiums an den Erwerb der Hochschulzugangsberechtigung in Form der allgemeinen Hochschulreife. Diese Verknüpfung von grundsätzlicher Berechtigung und faktischer Entscheidung zeigt sich exemplarisch anhand des folgenden Datenauszugs.

„Das war vorgegeben, mehr oder weniger von den Eltern. [...] Also das, das war nie so die Sache mit (.) die haben mir jetzt keine Uni ausgesucht, wie sie in der vierten Klasse mir, in der fünften Klasse mir das Gymnasium ausgesucht haben. Aber es war einfach, ja, du studierst. Es ist einfach so, eine GOTTgebene grundsätzliche Sache, du studierst. Das ist ja so, deswegen machst du ja überhaupt ein Abi.“ (P3-178-180)

In diesem Datenbeispiel dokumentiert sich über den Verweis auf den Einfluss der Eltern die Orientierung der Person an fremdinduzierten Erwartungen. Dieser Eindruck verstärkt sich noch dadurch, dass dieser Verbindung durch den Einbezug einer metaphysischen Instanz eine nahezu absolute Legitimität beigemessen wird. Etwas schwächer thematisiert dieses Phänomen auch P1: 
„Da war eigentlich, glaube ich, das war nicht die Frage, was man macht. [...] ich glaube, das ist einfach bei den Abimäusen so [...] Ja, waren wir so, was sollen wir sonst machen, also alle aus dem Klassenverband haben halt irgendwas studiert" (P1-149-151)

Dieser Auszug verdeutlicht aus der Perspektive der Betroffenen heraus die Selbstverständlichkeit bzw. Unhinterfragtheit der beschriebenen Kopplung. Die Anschlussfähigkeit dieses Phänomens zeigt sich im Vergleich mit empirischen Ergebnissen zum Übergang zwischen Schule und Arbeit. Dabei wird die Prägekraft gesellschaftlicher Erwartungen an Jugendliche herausgearbeitet, die sich in einem Übergangsregime manifestieren (Walther 2014: 91). Auch im Fall des Übergangs in die Hochschule wird dieses Phänomen deutlich, wobei die Personen ebenfalls Normalitäten konstatieren und eigene Entscheidungen tendenziell in den Hintergrund treten.

\section{Unterstützungskontexte beim Übergang in die Hochschule}

Auch vor dem Hintergrund von Fragen nach unterstützenden Kontexten, die die Subjekte zur Übergangsgestaltung in Anspruch nehmen, zeigen sich anschlussfähige Ergebnisse. In Ergänzung zu Ansätzen, den Übergang in die Hochschule mittels organisationaler Angebote zu rahmen (Driesen/Ittel 2019), verweisen die empirischen Daten auf eine Priorisierung lebensweltnaher Beratungskontexte. Deutlich wird dies anhand des nachfolgenden Auszugs:

„Ich sehe mich irgendwie noch, wie ich dieses Z4 Campusding wälze und nebenbei, ich glaube, mein Schwager und meine Schwester auch noch mit dabei saßen [...] Und gefragt haben, warum ich eigentlich so diesen Studiengang in R13 zum Beispiel, der irgendwie ich weiß gar nicht mehr warum der weggefallen ist, warum ich mich für den interessiere oder (.) ja allgemein meine Geschwister, die immer noch Input geliefert haben und überlegt haben, hey, wie wäre es denn noch damit" (P2-184-186)

Wie auch in weiteren Datenbeispielen scheint hier jenseits der Frage, ob organisationale Angebote in Anspruch genommen werden, vor allem das soziale Umfeld bei der Auswahl von Studienort und Studiengang relevant zu sein. Insbesondere dokumentiert sich in den analysierten Daten die deutliche Präferenz, sich im Entscheidungsprozess auf die Empfehlungen des sozialen Umfelds und hier vor allem der Familie sowie enger Freunde zu stützen. Demgegenüber werden Unterstützungskontexte von Hochschulen oder anderen Beratungseinrichtungen dethematisiert. Im Rahmen dieser spezifischen Konstellation beziehen sich die Studienteilnehmenden vor allem dann auf die Empfehlungen ihres sozialen Umfelds, wenn diese auf eigenen Studiererfahrungen beispielsweise 
von Geschwistern beruhen, die bereits ein Hochschulstudium abgeschlossen haben.

\section{Selbstbezogene Veränderungen im Kontext des Übergangs in die Hochschule}

Mit Blick auf die zweite Fragestellung und die theoretische Rahmung der transformatorischen Bildung lässt sich zunächst festhalten, dass im Zusammenhang mit der Aufnahme eines Hochschulstudiums keine Hinweise auf biografische Wandlungsprozesse (Schütze 1984: 92ff.) rekonstruiert werden konnten. Vielmehr zeigt sich, dass der Übergang in die Hochschule in den drei Fällen auf je unterschiedliche Weise gerahmt wird und spezifische Irritationen auftauchen, die als Anlässe für transformatorische Bildung gewertet werden können.

Die Erläuterungen einer Person verdeutlichen die Möglichkeit, mittels der Aufnahme eines Hochschulstudiums den multioptionalen Spielraum im mittleren Erwachsenenalter zu bewahren. So eröffnen sich durch diesen Schritt nicht nur neue berufliche Perspektiven, sondern es findet auch eine Erweiterung der sozialen Kontakte statt, die wiederum zu Irritationen des eigenen Selbst- und Weltverhältnisses führen kann. In diesem Zusammenhang dokumentiert sich zudem der Aspekt der Pfadabhängigkeit von Übergängen. Im konkreten Beispiel geschieht dies, indem durch die Familiengründung die Aufnahme eines Hochschulstudiums angeregt wird.

Aus der Perspektive einer anderen Person werden sowohl die Entscheidung für den gewählten Studiengang als auch der faktische Studienverlauf als krisenhafte Negativerfahrung konstruiert. Zwar wird von der befragten Person durchaus ein Zuwachs an spezifischem Fachwissen und bestimmten Kompetenzen diagnostiziert, doch führt der Verlauf des eigenen Studiums dazu, dass eine negativ konnotierte biografische Suchbewegung initiiert wird.

Auch die dritte Person konstatiert Fremdheitserfahrungen, die jedoch nicht auf die gesamte Studienphase bezogen werden. Stattdessen werden diese mit dem Hochschulwechsel nach Abschluss des Erststudiums verbunden (Hilgemann 2017: 59), die zusammen mit dem Aufbau eines als neu deklarierten sozialen Umfelds zu Irritationen führen. Sie dokumentieren sich in verschiedenen Phänomenen wie etwa der Rekontextualisierung retrospektiver Erfahrungen und prospektiver Zielsetzungen. Exemplarisch zeigt sich dies bei der Bewertung der eigenen Studienerfahrungen und der Ableitung von Handlungsempfehlungen für den eignen Nachwuchs zur zukünftigen Entscheidungsfindung. In diesem Zusammenhang finden sich starke reflexive Anteile, die überwiegend im Medium transgenerativer Projektionen realisiert werden. 


\section{Transformatorische Bildung im Kontext des Übergangs in die Hochschule}

In der Rückschau zeigen sich anhand der drei ausgewählten Fälle Phänomene, die einen Beitrag zur erziehungswissenschaftlichen Erforschung von Übergängen leisten können. Hinsichtlich der ersten Fragestellung nach den Dimensionen des Übergangs in die Hochschule lässt sich neben den gewählten Unterstützungskontexten vor allem die Existenz eines spezifischen Übergangsregimes zwischen weiterführender Schule und Hochschule rekonstruieren. Dieses manifestiert sich in den Sachverhaltsdarstellungen der befragten Personen primär in der Verknüpfung vom Erwerb der allgemeinen Hochschulreife und der Entscheidung für die Aufnahme eines Hochschulstudiums.

Mit Fokus auf die Bedingungen transformatorischer Bildung lassen sich zudem die simultanen Übergänge im Erwachsenenalter rekonstruieren. Diese scheinen im Datenmaterial am Beispiel des Übergangs in die Hochschule als Wohnortwechsel, Aufnahme einer Erwerbstätigkeit, Zusammenzug mit der*dem Partner*in, Familiengründung und der Kindererziehung auf. Hinsichtlich der zweiten Fragestellung nach selbstbezogenen Veränderungen in den Eigentheorien der Befragten konnten in den Interviews primär Anlässe für transformatorische Bildungsprozesse rekonstruiert werden. Dabei erwies sich vor allem die steigende Komplexität dieser simultanen Übergänge nicht nur aus theoretischer Perspektive, sondern auch mit Blick auf die empirischen Daten als Bedingung für potentiell krisenhafte Erfahrungen und damit als möglicher Anlass für transformatorische Bildungsprozesse. Während die Aufnahme eines Hochschulstudiums aus einer fallübergreifenden Perspektive dementsprechend genuin als ein solcher Anlass begriffen werden kann, zeigt sich mit Fokus auf die untersuchten Fälle ein differenziertes Bild. Dabei lässt sich ein Kontinuum an retrospektiven Einschätzungen feststellen, das von der Bestätigung des eigenen multioptionalen Spielraums durch den Übergang in die Hochschule einerseits bis hin zur Negativkonnotation des Studiums andererseits reicht.

\section{Literatur}

Autorengruppe Bildungsberichterstattung (2018): Bildung in Deutschland 2018. Ein indikatorengestützter Bericht mit einer Analyse zu Wirkungen und Erträgen von Bildung. Bielefeld: wbv. 
Driesel, Cornelia / Ittel, Angela (Hrsg.) (2019): Der Übergang in die Hochschule. Strategien, Organisationsstrukturen und Best Practices an deutschen Hochschulen. Münster: Waxmann.

Fisch, Stefan (2015): Geschichte der europäischen Universität. Von Bologna nach Bologna. München: Beck.

Hilgemann, Meike (2017). Der Übergang vom Bachelor zum Master. Bildungsentscheidungen im Schnittfeld von Gender und Fachkultur. Opladen: Budrich.

Hochschulrektorenkonferenz (2017): Statistische Daten zu Studienangeboten an Hochschulen in Deutschland. Studienangebote, Studierende, Absolventinnen und Absolventen. Wintersemester 2017/2018. Bonn: Hochschulrektorenkonferenz.

Hof, Christiane / Maier-Gutheil, Cornelia (2014): Übergänge im Erwachsenenalter. Befunde und offene Fragen für die Erwachsenenbildung. In: Hof, C. / Meuth, M. / Walther, A. (Hrsg.): Pädagogik der Übergänge. Übergänge in Lebenslauf und Biografie als Anlässe und Bezugspunkte von Erziehung, Bildung und Hilfe. Weinheim: Beltz Juventa, S. 146-167.

Koller, Hans-Christoph (2018): Bildung anders denken. Einführung in die Theorie transformatorischer Bildungsprozesse (2. Auflage). Stuttgart: Kohlhammer.

Middendorff, Elke/ Apolinarski, Beate / Becker, Karsten / Bornkessel, Philipp / Brandt, Tasso / Heißenberg, Sonja / Poskowsky, Jonas (2017): Die wirtschaftliche und soziale Lage der Studierenden in Deutschland 2016. 21.Sozialerhebung des Deutschen Studentenwerks durchgeführt vom Deutschen Zentrum für Hochschul- und Wissenschaftsforschung. Bonn /Berlin: Bundesministerium für Bildung und Forschung.

Schäffter, Ortfried (2014): Navigieren durch vernetzte Bildungslandschaften. Zum impliziten Erwerb von Übergangskompetenz in Lernbiographien. In: Felden, H. v. / Schäffter, O. / Schicke, H. (Hrsg.): Denken in Übergängen. Weiterbildung in transitorischen Lebenslagen. Wiesbaden: Springer VS, S. 37-59.

Schäffter, Ortfried (2015): Übergangszeiten - ,Transitionen' und ,Life Trajectories'. Navigieren durch Bildungslandschaften im Lebensverlauf. In: SchmidtLauff, S. / Felden, H. v. / Pätzold, H. (Hrsg.): Transitionen in der Erwachsenenbildung. Gesellschaftliche, institutionelle und individuelle Übergänge. Opladen: Budrich, S. 19-34.

Schütze, Fritz (1984): Kognitive Figuren des autobiographischen Stegreiferzählens. In: Kohli, M. / Robert, G. (Hrsg.): Biographie und Soziale Wirklichkeit: neue Beiträge und Forschungsperspektiven. Stuttgart: Metzler, S. 78-117.

Statistisches Bundesamt (2018a): Bildung und Kultur. Studierende an Hochschulen. Wintersemester 2017/2018. Wiesbaden: Statistisches Bundesamt.

Statistisches Bundesamt (2018b): Entwicklung der Studienanfängerquote* in Deutschland von 2000 bis 2017. https:/de.statista.com/statistik/daten/studie/ 72005/umfrage/entwicklung-der-studienanfaengerquote/ [Zugriff 22.09.18].

Strübing, Jörg (2014): Grounded Theory. Zur sozialtheoretischen und epistemologischen Fundierung eines pragmatistischen Forschungsstils (3. Auflage). Wiesbaden: VS. 
Walther, Andreas (2014): Übergangsregimes. Übergänge zwischen Schule und Arbeit im internationalen Vergleich. In: Hof, C. / Meuth, M. / Walther, A. (Hrsg.): Pädagogik der Übergänge. Übergänge in Lebenslauf und Biografie als Anlässe und Bezugspunkte von Erziehung, Bildung und Hilfe. Weinheim: Beltz Juventa, S. $78-103$.

Witzel, Andreas / Reiter, Herwig (2012): The Problem-Centred Interview. Los Angeles: SAGE. 